\title{
" Il seno nudo », ou Palomar et les derniers avatars de la scène de séduction
}

Anne Boulé-Basuyau

\section{OpenEdition}

Édition électronique

URL : http://journals.openedition.org/cei/845

DOI : $10.4000 /$ cei.845

ISSN : 2260-779X

Éditeur

UGA Éditions/Université Grenoble Alpes

Édition imprimée

Date de publication : 15 septembre 2006

Pagination : 267-278

ISBN : 978-2-84310-086-4

ISSN : 1770-9571

\section{Référence électronique}

Anne Boulé-Basuyau, « « II seno nudo », ou Palomar et les derniers avatars de la scène de séduction », Cahiers d'études italiennes [En ligne], 5 | 2006, mis en ligne le 15 mars 2008, consulté le 26 mars 2021. URL : http://journals.openedition.org/cei/845 ; DOI : https://doi.org/10.4000/cei.845 


\title{
«IL SENO NUDO», OU PALOMAR \\ ET LE DERNIER AVATAR DE LA SCÈNE DE SÉDUCTION
}

\author{
Anne Boulé-Basuyau \\ Université de la Sorbonne nouvelle - Paris 3
}

Chez un écrivain comme Italo Calvino qui, en quarante ans de production littéraire, n'a jamais choisi une femme pour protagoniste d'un de ses romans, et qui, en 1960, en repensant à l'époque de la rédaction du Sentiero dei nidi di ragno, déclarait déjà: "je n'étais pas intéressé - et peutêtre n'ai-je guère changé depuis lors - par la psychologie, l'intériorité $[\ldots] »^{1}$, on n'est guère surpris que le thème de la séduction, propice à l'introspection et à l'évocation des mouvements de l'âme, soit si peu présent dans son œuvre.

Les rares occasions où l'écrivain s'est inspiré de ce topos narratif n'en prennent que plus de relief, d'autant qu'elles sont l'un des lieux de l'expression de la "comicità" calvinienne. Parmi celles-ci, nous avons choisi d'analyser "Il seno nudo", publié par Calvino dès 1977 dans Il Corriere della Sera et repris en 1983 dans le recueil Palomar avec quelques modifications $^{2}$.

Après avoir montré en quoi ce texte se rattache au thème romanesque de la séduction et comment Calvino se singularise dans son traitement, nous le mettrons en parallèle avec une production plus ancienne de l'auteur, dont il semble être le pendant moderne: le fameux épisode de

1. In «Postfazione ai Nostri antenati. (Nota 1960)». Comme l'analyse D. Ferraris, "très tôt [...] l'ennemi à combattre a pu être clairement désigné sous un vocable simple: l'émotion» ("Italo Calvino: l'Ordre et la Chair», Revue des Études italiennes, tome 47, nº 3-4, juil-déc 2001, p. 213).

2. Le texte paraît en $1^{\mathrm{e}}$ page du Corriere della sera du 2 août 1977 , sous le titre originel: «Un uomo e un seno nudo all'orizzonte». La version publiée dans le volume Palomar échange le protagoniste anonyme, "un uomo ", contre Palomar et le titre se réduit désormais au seul syntagme «Il seno nudo». 
l'«innamoramento » de Rambaldo, dans Il cavaliere inesistente. En mettant en évidence constantes et variantes, la lecture croisée des deux textes, produits à dix-huit ans d'intervalle, permettra de mieux saisir l'interprétation que Calvino propose de ce thème dans la deuxième moitié du XXe siècle.

\section{La séduction dans «Il seno nudo»}

Dominé par la figure du protagoniste, Palomar est une œuvre où les personnages féminins sont très rares. L'épouse de Palomar jardine à ses côtés dans «Il fischio del merlo»; dans «La pancia del geco», tous deux observent l'animal qui a coutume, le soir, de venir se chauffer sur la vitre extérieure de leur vitrine; dans aucun de ces deux microtextes, cette épouse n'apparaît comme un objet érotique. Dans "Gli amori delle tartarughe», Palomar épie l'accouplement de deux tortues mais, réduit à une lente poursuite de la femelle par le mâle puis à un frottement de deux carapaces, il n'éveille chez le protagoniste qu'une perplexité et une curiosité d'ordre scientifique. Seul «Il seno nudo", qui met en scène une jeune inconnue en monokini, introduit un personnage féminin dans sa dimension érotique, laquelle induit l'apparition du motif de la séduction.

Placé dans le premier chapitre des "Vacanze di Palomar», qui le situe "Sulla spiaggia», l'épisode succède à l'observation des vagues et précède celle des reflets du soleil couchant. Calvino y décline diverses réactions masculines face à la poitrine nue d'une femme, en fonction des préjugés de l'homme, de son respect pour la Femme, de son souci de discrétion, mais aussi en fonction de "l'air du temps", à une époque où la libération des mœurs - rappelons que le texte est publié la première fois en 1977 commence à se traduire par l'abandon du soutien-gorge, en particulier sur les plages.

Comme l'explique l'écrivain dans le «mode de lecture» qu'il livre avant la table des matières, la numérotation qu'il a affectée à cet épisode indique que des éléments anthropologiques y sont présents et que le texte s'organise principalement sous la forme d'un récit. En effet, Palomar se promène sur la plage quand son attention est attirée par une femme qui prend le soleil, seins nus; la question que se pose Palomar, "uomo discreto" troublé par cette vision, est de savoir quelle qualité de regard il convient d'adresser à cette nudité. Parce que la réponse est difficile, Palomar modifie le déroulement de sa promenade: il passe et repasse devant la jeune femme, au rythme de ses interrogations. Le texte s'organise en quatre parties qui correspondent aux quatre passages de l'homme devant elle, cha- 
cun d'eux se caractérisant par un certain type de regard. Du refus de voir initial $^{3}$ - motivé par un profond respect pour la Femme -, Palomar passe, après deux étapes intermédiaires ${ }^{4}$, au regard délibérément appuyé de la fin, qui veut rendre ouvertement hommage à cet attribut de la féminité. S'il y a quatre passages devant la femme, c'est-à-dire quatre types de regard, c'est parce qu'aucun ne le satisfait durablement. À chaque passage, le doute s'insinue quant à la légitimité du regard choisi, amenant le protagoniste à faire demi-tour pour expérimenter une autre façon de regarder cette poitrine, plus respectueuse - pense-t-il - de la personne, de son sexe: un regard en somme apparemment plus libre de préjugés que le précédent. Le texte est donc construit à partir d'un schéma très simple: adoption d'un type de regard / satisfaction passagère / doute sur la légitimité de ce regard. Le schéma est reproduit trois fois intégralement. Mais, lors du quatrième passage de Palomar, avant que le doute ne survienne de nouveau, la procédure est interrompue par l'objet même de l'observation, qui devient alors sujet agissant. "[...] Lei si alza di scatto, si ricopre, sbuffa, s'allontana [...]" : ce qui met un terme définitif aux interrogations de Palomar, à ses allées et venues et à l'épisode.

Deux inconnus de sexe opposé, quasiment seuls sur une plage: c'est une situation presque banale à partir de laquelle un écrivain peut aisément imaginer une entreprise de séduction. Du reste, puisque la promenade originellement linéaire se métamorphose en de brefs et répétitifs allers et retours ${ }^{6}$, et que "séduire» signifie étymologiquement "détourner du chemin», Palomar, littéralement aimanté par la jeune femme aux seins nus, est effectivement séduit par elle. Mais on a affaire à un curieux épisode de séduction, Calvino battant en brèche les principaux stéréotypes que véhicule ce thème en littérature.

Le topos de la séduction comme jeu à deux, opposant un gagnant - le séducteur conquérant (presque toujours de sexe masculin) - et un perdant- le plus souvent, la femme soumise à l'entreprise de séduction - est refusé par Calvino, et même inversé, dans «Il seno nudo»: ici, la séduc-

3. "Palomar, uomo discreto, volge lo sguardo all'orizzonte marino".

4. Lors de ces deux nouveaux passages, Palomar décide d' "effleurer» le sein de son regard: le regard embrasse tout d'abord indifféremment tous les éléments du paysage, y compris cette poitrine; puis, la fois suivante, parce qu'il sent qu'il doit montrer qu'il reconnaît le statut particulier de ce sein, Palomar salue par « uno scarto, quasi un guizzo » l'entrée de la poitrine dans son champ visuel.

5. "Ora il suo sguardo [...] si soffermerà sul seno con uno speciale riguardo".

6. Chacune des quatre parties s'ouvre précisément par un verbe de mouvement: "cammina", "Ritornando/ripassa", "Si volta e ritorna», "Fa dietro-front». 
tion émane de la femme et c'est l'homme qui a le rôle passif du partenaire séduit. Mais il ne faut pas y voir pour autant une concession de la part de l'auteur aux revendications égalitaires de type féministe, vives en Italie à partir du milieu des années $70^{7}$, car cette femme, qui se croyait tranquille sur la plage isolée, n'a rien d'une conquérante, et la fin du texte confirme qu'elle se serait bien passée de l'intérêt que lui porte Palomar. Quant à celui-ci, absorbé par les cogitations où le plonge la vue de cette poitrine nue, il est loin de reconnaître le plaisir que cette vision a fait naître en lui et qui reste masqué par les opérations mentales auxquelles il s'adonne sans fin. Les différentes façons par lesquelles ce sein est nommé - «nuvola bronzeo-rosea », «ricolma luna di pelle più chiara», "cuspidi aureolate»l'attestent: le recours à la métaphore et à un lexique recherché permet une mise à distance de l'objet érotique qui a éveillé la fascination masculine ${ }^{8}$. Dans "Il seno nudo", Calvino imagine donc une séduction d'un genre nouveau puisque, d'un côté, il n'y a pas intention de séduire et, de l'autre, pas de conscience d'être séduit.

On remarque également que le thème de la séduction, tout en occupant l'épisode entier, est réduit au moment appelé en littérature «scène de séduction ": il n'y a ni un avant (où serait évoqué l'état de manque dont souffrirait l'un des personnages, ou l'esprit de conquête chez un autre, les prédisposant respectivement à être séduit et à séduire), ni un après (constitué des suites psychologiques de la rencontre, du développement de la relation née de cette rencontre). Dans ce texte, le thème de la séduction s'exprime strictement dans le face à face momentané de deux inconnus. Encore qu'il soit même exagéré de parler de "face à face», les approches de Palomar étant plus latérales que frontales et la rencontre se réduisant plutôt au passage de l'un devant l'autre, sans que l'on sache, sauf dans les dernières lignes, ce qu'en pense précisément l'autre.

Cette autre a d'ailleurs une présence textuelle fort restreinte: il n'y a pas de description de la femme observée, alors que la «scène de première vue»" est très souvent l'occasion d'un portrait féminin, y compris à travers

7. Rappelons que la première grande manifestation du mouvement féministe en Italie eut lieu à Rome en décembre 1975; elle rassembla environ 20000 participants. La même année, le Movimento di liberazione delle donne italiane recueillit 800000 signatures pour l'organisation d'un référendum sur l'avortement.

8. Ce phénomène relève de la mise à distance que $\mathrm{D}$. Ferraris décèle dans tout l'œuvre de Calvino et qu'il relie au besoin de maîtrise du monde par l'écrivain, «[...] une mise à distance de toute matière susceptible d'être un objet de représentation artistique ou littéraire, à commencer par les pulsions et désirs humains qui constituent par excellence le danger du dérèglement majeur" (art. cit., p. 218).

9. L'expression dérive du titre de l'excellent essai de Jean Rousset, Leurs yeux se rencontrèrent. La scène de première vue dans le roman, Paris, José Corti, 1984. 
le topos de la beauté indicible. Le corps de la jeune femme n'existe dans le texte de Calvino que de façon métonymique: par sa seule poitrine. Et celle-ci, pourtant vecteur de la séduction, n'est décrite que succinctement, à l'aide de qualificatifs génériques: "petto [...] fresco e piacevole alla vista".

On n'est guère surpris, dans ces conditions, de constater une autre infraction au déroulement de la scène de séduction classique: alors que le concept de séduction est lié à l'échange entre deux êtres qui pourraient devenir deux partenaires, pas un mot n'est prononcé dans «Il seno nudo ». Et, du moins jusqu'au dénouement, le regard ne semble qu'à sens unique: c'est celui de l'homme sur la femme. Bien qu'aucune réciprocité ne l'y encourage, Palomar continue imperturbablement ses allées et venues sous le nez de la jeune femme et son regard, selon la gradation déjà évoquée, se focalise de plus en plus sur cette poitrine. Dernier paradoxe, qui sert de conclusion abrupte à la scène de séduction: l'homme séduit est finalement pris pour un séducteur de bas étage, « un satiro » qui fait fuir la jeune femme.

Par le traitement anti-conventionnel, en particulier anti-sentimental et anti-romanesque, qu'il inflige au thème de la séduction dans Palomar, Calvino se positionne de façon originale dans l'histoire de la littérature. Mais le détournement des stéréotypes liés au thème de la séduction, observé dans "Il seno nudo", n'est pas un cas unique dans son œuvre. Il existe un précédent à cette scène de séduction hors norme: l'épisode de l'«innamoramento» de Rambaldo au chapitre IV du Cavaliere inesistente.

\section{Autre «scène de première vue"}

Les différences entre les deux œuvres sont nombreuses: le dernier volume de la trilogie pseudo-historique est un court roman tissé de réminiscences littéraires, une parodie du genre chevaleresque, un texte riche en rebondissements et en personnages invraisemblables; Palomar est au contraire un recueil de proses brèves empreintes de méditation, une œuvre de la maturité de l'écrivain, plus proche de l'essai que d'un véritable roman, dominée par un unique personnage proposé comme un alter ego de l'auteur. Mais, en dépit de ces différences, les passages qui nous intéressent, construits tous deux autour de la rencontre d'un homme et d'une femme, déclinent le thème de la séduction selon des modalités comparables. 
Les situations initiales sont très proches en ce qu'elles mettent en scène une femme épiée par un homme dans un moment d'intimité: l'héroïne du roman de 1959 est en train d'uriner, la jeune femme des années 70 prend le soleil sur la plage. Dans les deux cas, l'homme, métamorphosé en voyeur, est confronté à une semi-nudité féminine. D'un roman à l'autre, Calvino inverse simplement la partie dénudée: partie inférieure du corps pour la guerrière du temps de Charlemagne, partie supérieure pour la femme du XXe siècle. Réduite à l'un de ses attributs sexuels, la femme devient un objet d'observation ô combien troublant, même si ce trouble s'exprime de façon différente dans les deux romans. Le jeune Rambaldo, envahi par la stupéfaction et l'émotion, est immédiatement séduit: «Ne fu tosto innamorato"; tandis que Palomar, homme d'âge mûr et marié de surcroît, veut ignorer ce trouble et se réfugie dans ses cogitations. En un premier temps inconsciente du regard du bachelier, la guerrière est comme la jeune femme moderne dont elle partage l'anonymat - une séductrice involontaire. Les deux épisodes mettent donc en scène le même décalage entre le désir masculin, éveillé par le spectacle d'une femme séductrice malgré elle, et la réalité: l'unilatéralité de ce désir.

Les deux scènes connaissent la même issue immédiate: la femme, enfin consciente du regard indiscret de l'homme et de son désir, s'éloigne, exaspérée. La réaction de la guerrière - "tratto dalla cintola un pugnale glielo tirò contro »-, liée à sa pratique virile des armes, préfigure sur un mode exacerbé la réaction de la baigneuse, limitée, en ces temps moins belliqueux, à quelques "scrollate infastidite delle spalle». Ainsi toutes deux rompent-elles brutalement la fascination masculine en laissant sur le terrain, respectivement, un Rambaldo "vergognoso" et un Palomar amer de ne pas avoir été compris: dans les deux cas, un homme frustré.

En somme, bien que les deux romans different de façon flagrante, on est frappé par les constantes qui se dégagent de l'examen des deux textes. La scène de première vue semble avoir été conçue dans "Il seno nudo" comme une sorte de pendant de celle du chapitre IV du roman de 1959, puisqu'elle en reprend, dix-huit ans plus tard, les traits essentiels: le schéma de ces deux scènes de séduction est le même, le rapport homme/femme est similaire, l'issue identique et le traitement est humoristique dans les deux cas. Toutefois, le comique, capital dans les deux épisodes, fonctionne de façon très différente. 


\section{Le comique dans les scènes de séduction}

On ne doit guère être surpris que la "comicità" calvinienne s'exprime dans ces scènes de séduction. Déjà en 1969, dans «Il sesso e il riso ", l'écrivain rappelait " [...] le lien profond, au niveau anthropologique, entre le sexe et le rire». Les deux épisodes l'illustrent bien, tout en exploitant des formes de comique dissemblables.

Dans le passage du Cavaliere, le sourire du lecteur naît du cumul de divers procédés. Le comique de cet épisode où est révélée abruptement l'identité féminine de l'inconnu et qui, de ce fait, peut se métamorphoser en scène de séduction, résulte du décalage entre un registre soutenu et la trivialité de la situation narrée, ainsi que du détournement de plusieurs hypotextes ${ }^{10}$. Le dévoilement de la féminité du sauveur s'inspire de la scène du double coup de foudre, au Livre III de l'Orlando innamorato (Chant V). Calvino reprend la situation centrée sur la guerrière, mais en inversant les termes de la situation: Roger est remplacé par Rambaldo, le retrait du casque par celui de la partie inférieure de l'armure, et le dévoilement volontaire de Bradamante par un exercice de voyeurisme masculin. C'est en fait la situation littéraire traditionnelle qui est parodiée, la contemplation du visage féminin y étant remplacée par la vision volée de la moitié inférieure dénudée du corps féminin. La conclusion de l'épisode, "Rambaldo ne fu tosto innamorato", couronne cette scène de parodie comique: Rambaldo est séduit, et même conquis, comme l'avait été Roger, mais au terme d'une libre transposition des étapes intermédiaires, où la miction apparaît comme un élément fonctionnel inédit d'une scène de séduction d'un genre nouveau ${ }^{11}$.

Dans le volume Palomar, qui met en scène l'homme contemporain, les effets comiques de décalage entre la matière narrée et le registre de langue étant plus limités, Calvino s'en remet à d'autres procédés. Dans ce recueil

10. Sur les sources dont Calvino s'est inspiré pour ce roman, voir notre article: «Calvino et la littérature chevaleresque: Pulci, Boiardo, l'Arioste et les autres... dans Il cavaliere inesistente», in Diffusion et réception du genre chevaleresque, Presses de l'Université de Toulouse-Le Mirail, Collection l'ÉCRIT nº10, 2005, p. 269-293.

11. Si la fin insolite de cette scène de séduction, suivie de rapides ablutions, n'est inspirée par aucun texte antérieur, elle sert en revanche quelques années plus tard d'hypotexte à Calvino luimême, qui la transpose, en un style un peu moins élevé, dans le chapitre «Storia del guerriero sconosciuto" de La taverna dei destini incrociati. Mais dans cette nouvelle version, où l'on retrouve pourtant, plus directement, l'influence de Boiardo, dans la chevelure libérée par le retrait du heaume, le chevalier-voyeur ne s'abandonne pas à la contemplation de la féminité révélée du guerrier inconnu - en somme, l'épisode ne tourne pas à la scène de séduction: l'homme, furieux d'avoir été défait par une femme, lui vole son épée et prend la fuite. 
à la structure sérielle, "Il seno nudo" est lui-même construit de façon sérielle, ce qui engendre un comique de répétition. Les choix lexicaux et rhétoriques concourent eux aussi au sourire du lecteur: l'oxymore «reggipetto mentale" génère un évident comique de mots. La chute de l'épisode où l'homme, convaincu de sa discrétion, est pourtant comparé à un satyre, relève du phénomène humoristique baptisé par certains critiques «malice des objets». Il désigne tout ce qui s'oppose à la volonté de savoir et de comprendre du sujet: caractère insaisissable des vagues, foule de touristes japonais qui fait obstacle à sa méditation, etc. Ici, la "malice des objets", ce sont les préjugés contre lesquels Palomar essaye de lutter, pour rendre au sein sa dignité; c'est aussi la réaction de la femme, objet de l'observation, qui brusquement se rebelle en se méprenant sur les "intenzioni più illuminate» de Palomar. Ce sont en somme les préjugés de la femme conjugués à ceux du protagoniste. Enfin, on ne peut manquer de sourire de l'écho franciscain de l'antépénultième paragraphe: "Il suo sguardo [...] s'affretterà a coinvolgerlo [il seno] in uno slancio di benevolenza e gratitudine per il tutto, per il sole e il cielo, per i pini ricurvi e la duna e l'arena e gli scogli e le nuvole e le alghe, per il cosmo che ruota intorno a quelle cuspidi aureolate». Le cantique de saint François, "Laudes creaturarum", y est humoristiquement détourné: l'amour pour toutes les choses finit par englober cette poitrine nue qui, sacralisée par la métaphore des "cuspidi aureolate», devient même le nouveau centre du cosmos, se substituant en cela à l' "altissimu, onnipotente, bon Signore» à qui François rendait hommage dès le premier vers. Après cette évocation de l'amour universel, envolée poétique d'un Palomar heureux d'avoir enfin trouvé le bon regard, la chute n'en est que plus dure: l'homme pétri de poésie primitive est rabaissé au rang de satyre importun.

\section{Fonctions de la scène de séduction}

$\mathrm{Si}$, dans chacun des deux passages, la scène de séduction est l'occasion de faire sourire le lecteur, sa fonction, au niveau du livre, ne se limite évidemment pas à cela. Dans Il cavaliere, l'amour, né dans le cœur de Rambaldo au cours de cet épisode, introduit un élément dynamique dans le récit, réactive le binôme topique de la littérature chevaleresque "l'arme, gli amori», dont Calvino donne cette interprétation personnelle: "La guerra la combatti bene soltanto dove tra le punte delle lance intravedi una bocca di donna, e tutto, le ferite il polverone l'odore dei cavalli, non ha sapore che di quel sorriso" (chap. VII). Outre le plaisir jubilatoire 
qu'elle procure au lecteur averti, et qui tient à la connivence que suppose tout jeu d'hypertextualité, la scène de séduction est donc également fonctionnelle au niveau de la trame, puisqu'elle transforme le jeune héros, lui donne un but - se faire aimer de la belle guerrière -, ce qui l'implique dans une série de quêtes et de poursuites qui déboucheront sur la réciprocité de cet amour. La scène de première vue du chapitre IV, qui s'était conclue par le geste de colère et la fuite de la jeune femme, connaît en fait une seconde issue, issue retardée mais heureuse: au chapitre XII, Bradamante, enfin convaincue de la valeur et de l'amour du jeune Rambaldo, s'abandonne elle aussi à cette passion et l'avenir s'ouvre à eux.

Nulle réciprocité en revanche dans "Il seno nudo", pas de "lieto fine" à l' «aventure » de Palomar sur la plage, qui reste purement visuelle et cérébrale et se clôt dans les limites exactes du microtexte. Loin d'apporter un regain de vitalité au protagoniste et d'entraîner des conséquences sentimentales sur son quotidien d'homme d'âge mûr et déjà marié, la scène de séduction est un épisode visant à accroître son isolement. Elle consacre l'échec de la tentative de communication par le regard ${ }^{12}$ et la faillite de toute volonté de compréhension mutuelle. Il n'y a pas de partage possible dans l'univers de Palomar, encore moins de communion entre les êtres.

Naturellement, lorsque le texte fut publié pour la première fois, en page 1 du Corriere della Sera du 2 août 1977, sous la rubrique "Si diffonde, anche se fioccano le denunce, la moda del bikini», l'épisode avait une valeur et une fonction autres. En page 2 du même quotidien, dans un article intitulé «Operazione-lampo della polizia a Cesenatico. Denunciate cinque ragazze con il monokini", Vittorio Monti racontait sur un mode plaisant les récentes mésaventures de cinq belles Italiennes surprises seins nus sur une plage romagnole et dûment verbalisées par les représentants de la force publique, eux-mêmes en maillot de bain. Le récit de cette «[...] retata in difesa della pubblica decenza" permettait au journaliste de rappeler les sanctions pénales encourues. Le lecteur pouvait s'attendre à ce que l'article de Calvino, publié dans ce contexte, c'est-à-dire dans les pages de ce même numéro du Corriere della Sera, présente le point de vue de l'intellectuel sur ce nouveau fait de société. Mais dans «Un uomo e un seno all'orizzonte", l'intérêt de Calvino se porte sur l'effet produit par une poitrine dénudée sur celui qui la regarde. Au lieu de se positionner explicitement face à ce problème de société qui déchaîne à l'époque les passions, l'écrivain invente une petite nouvelle mettant en scène «una gio-

12. Ailleurs dans le livre, c'est l'échec de la communication verbale qui est le plus souvent illustré. 
vane donna $[\ldots]$ distesa sull'arena $[\ldots]$ a seno nudo" et un "uomo, persona civile e discreta»; à travers le trouble et les interrogations du personnage masculin, Calvino invite le lecteur du quotidien à réfléchir plus en profondeur sur ce phénomène ${ }^{13}$, tout en lui offrant, grâce au ton et à la chute du texte, une occasion de s'amuser en lisant - chose appréciable en pleines vacances d'été. Sous un abord divertissant, la petite fiction imaginée par Calvino est la réponse à la fois posée et spirituelle de l'intellectuel à l'excessive effervescence suscitée par ce problème de société ${ }^{14}$.

Lorsque, six ans plus tard, ce récit, à peine remanié au plan de l'écriture mais profondément transformé par l'introduction de "Palomar» (qui remplace désormais l'inconnu de 1977), rejoint les autres articles publiés au cours des années précédentes, pour s'organiser en recueil, l'épisode prend une autre valeur et une autre résonance. L'évolution des mours dont témoignait l'article, en mettant en scène une femme désireuse de disposer de son corps comme bon lui semble, ne parait plus représenter l'essentiel du microtexte, son noyau, mais plutôt une sorte d'arrière-fond, comme ailleurs dans le livre la terrasse ou le zoo, qui sont simplement les éléments d'un décor où se déroule la série des «aventures» de Palomar. La contextualisation nouvelle de l'épisode, désormais inséré dans un livre qui constitue un projet et un enjeu intellectuel tout autre, fait que le microtexte, naturellement toujours centré sur la scène de séduction, acquiert un autre sens: il devient une étape dans le processus d'anéantissement progressif du protagoniste: pris pour un satyre à la fin de "Il seno nudo", Palomar est ensuite considéré comme "demente» par la petite foule qui l'observe avec perplexité dans la conclusion de «La contemplazione delle stelle», puis comme "debol[e] di mente» par les clients de la fromagerie dans les dernières lignes du "Museo dei formaggi». "Il seno nudo" et sa scène de séduction contribuent en fait à l'entreprise de négation systématique des efforts du protagoniste pour comprendre le monde qui l'entoure et y trouver sa place. En écrivant que "Chaque microtexte coïncide [...]

13. V. Spinazzola rappelle, à propos de l'implication de Calvino face à des sujets d'actualité, sa «[...] predilezione insistita per la stesura del bilancio di una somma di dati d'esperienza, a suffragio della volontà di giungere a un chiarimento concettuale e valutativo spassionato " (" L'io diviso di Italo Calvino", in Italo Calvino. Atti del convegno internazionale Firenze 26-28 febbraio 1987, ouvrage collectif, édition établie par G. Falaschi, Milan, Garzanti, 1988, p. 110). Car la mission de l'intellectuel, selon Calvino, n'est pas d'imposer des solutions ou des vérités toutes faites, mais de guider le lecteur dans l'ardu décodage de la réalité complexe qui l'entoure.

14. Comme l'écrit encore Spinazzola, «Le manifestazioni di novità, le infatuazioni o nevrosi collettive eccitavano in lui una risposta di pacatezza, come di chi bada a capire e far capire, ossia aiuta i lettori a superare gli stati emotivi per orientarsi equilibratamente nella realtà" (op.cit., p. 109). 
avec l'étape d'un voyage vers le vide ${ }^{15}$ ", Claudio Milanini a exprimé fort efficacement la progression de Palomar vers le néant. Au fil des microtextes, de façon plus sensible dans la troisième partie intitulée «I silenzi di Palomar», le dénouement se dessine peu à peu, inévitable: l'explicit "In quel momento muore", on ne peut plus laconique, sanctionne définitivement la vanité des tentatives cognitives du protagoniste en mettant un terme à la reproduction sérielle de ses aventures.

L'évolution entre les scènes de séduction des deux œuvres, pour ce qui concerne l'image et le destin de leur protagoniste masculin respectif et la représentation des rapports entre homme et femme, témoigne d'un pessimisme croissant de la part de l'écrivain, on l'aura compris. Au début du Cavaliere inesistente, Rambaldo était certes inférieur, dans le domaine du maniement des armes, au personnage féminin doté de vertus militaires au contraire très affirmées; on peut y voir du reste une interprétation calvinienne de la crise de la virilité observée dans la littérature européenne depuis le XIX siècle. Mais le jeune héros, au terme du roman, n'en finissait pas moins par conquérir Bradamante à force de ténacité et de constance: l'homme retrouvait ainsi un statut de digne partenaire ${ }^{16}$.

Dans Palomar, l'évolution des mœurs qui sert de toile de fond au microtexte n'est qu'une illusion de progrès: elle ne conduit pas, dans la situation décrite sur la plage, à une plus grande harmonie entre les êtres de sexes différents. Les préjugés sortent au contraire renforcés de cette scène de première vue, le fossé entre les sexes est confirmé, la solitude de Palomar plus évidente et plus inéluctable. Ce dernier avatar de la scène de séduction, déclinée sur un mode qui déçoit toute attente sentimentale chez le lecteur, participe de la destruction du romanesque et, concrètement, du personnage masculin dans l'œuvre de Calvino. Dans cet épisode de son dernier "roman", l'écrivain consacre l'inévitable infériorité de l'homme, face à une femme qui, en refusant son regard et en quittant les lieux, décide tout bonnement de se passer de lui. Cette fin, que l'on peut qualifier de castratrice, préfigure la «vraie» mort du personnage fictif à la

15. Milanini C., L'utopia discontinua, Milan, Garzanti, 1990, p. 173.

16. Cette sorte de réhabilitation du personnage dans le domaine érotique, précédée de sa réhabilitation dans le métier des armes, contribue à faire de Rambaldo le protagoniste de l'œuvre; le jeune homme vole en effet ce statut au personnage éponyme du roman qui, malgré sa volonté à exister, ne peut, par exemple, satisfaire les exigences sexuelles de la belle Priscilla au chapitre VIII, si ce n'est, faute de mieux, par les artifices d'un discours érotique remplaçant les actes par les mots. 
fin du livre. Ultime incursion de Calvino dans l'univers littéraire de la séduction, "Il seno nudo" achève de façon emblématique son itinéraire toujours prudent au pays d'Eros. 